\title{
Entre exception et ordinaire ${ }^{1}$
}

\author{
Luigi Achilli et Lucas Oesch
}

$\mathrm{E}$ $\mathrm{n}$ 2015, il y avait plus de 5 millions de réfugiés palestiniens enregistrés auprès de l'UNRWA (Office de secours et de travaux des Nations unies pour les réfugiés de Palestine dans le Proche-Orient) et répartis entre la Cisjordanie, la Bande de Gaza, la Jordanie, la Syrie et le Liban (UNRWA 2015). Parmi ceux-ci, plus de 1.5 million, ou près de $30 \%$, habitent dans des camps.

En s’inspirant des travaux de Giorgio Agamben (1997, 2003), la littérature scientifique sur les camps de réfugiés les a souvent assimilés à des " espaces d'exception " à l'intérieur desquels la population se retrouverait dans une position de " vie nue ", une vie d'exclusion par définition, soumise à la seule volonté du pouvoir souverain. La répartition des réfugiés à l'intérieur d'un espace conçu comme homogène - le

1 Cet ouvrage constitue la publication des actes d'un colloque intitulé « Palestinian Refugee Camps in Jordan and the Near East : Living, Planning and Managing a Lasting Temporariness " qui s'est tenu le 7 mars 2011 à Amman (http ://www.ifporient. org/node/873). Nous remercions le Service de Coopération et d'Action Culturelle de l'Ambassade de France en Jordanie, l'Institut français du Proche-Orient (IFPO) et le Council for British Research in the Levant (CBRL) de leur soutien à l'organisation du colloque, ainsi que l'IFPO de sa contribution à cette publication. Nous tenons également à remercier les relecteurs externes qui ont consacré une partie de leur temps précieux à cet ouvrage, ainsi que le comité de rédaction de A contrario. camp - est un dispositif utilisé à la fois par les pouvoirs étatiques et les acteurs humanitaires afin de contrôler ce qui est entrevu comme un "surplus d'humanité " (Malkki 1995 : 498). Dans de tels espaces, la vie des individus est perçue, accommodée, contrôlée et disciplinée à travers des catégorisations humanitaires (réfugié, personne vulnérable, etc. : voir par exemple Agier 2008; Daniel et Knudsen 1995 ; Harrell-Bond 1999 ; Hyndman 2000 ; Long 1993 ; Low et Lawrence-Zuniga 2002 ; Malkki 1995, 1997 ; Soguk 1999). La généalogie de cette pensée sur les camps peut être reliée à la théorisation de Hannah Arendt sur les réfugiés en tant qu'une " anomalie ». Cependant, c'est certainement les travaux d'Agamben qui fournissent la tentative la plus récente de conceptualisation des camps (1997, 2003). En s’inspirant de la pensée de Carl Schmitt, Agamben 
remet en question l'analyse du pouvoir étatique moderne proposée par Michel Foucault. D’après Foucault, ce pouvoir est exercé par la discipline et le contrôle de sujets libres. Pour Agamben, le pouvoir de l'État repose sur la possibilité de réduire les individus à une " vie nue ", à savoir des individus dépouillés de leurs statuts et droits politiques, auxquels il ne reste qu'une vie biologique. Cet " état d'exception " est instauré par le souverain qui définit les limites de la loi et décide de sa suspension, en tant qu'une " nécessité " résultant d'une menace pesant sur l'ordre politique. Dans ce cadre, le camp de réfugiés apparaît comme une matérialisation de l'état d'exception :

"Le camp est l'espace qui s'ouvre lorsque l'état d'exception commence à devenir la règle. L'état d'exception, qui était essentiellement une suspension temporelle de l'ordre juridique sur la base d'une situation réelle de danger, acquiert désormais, dans le camp, une assise spatiale permanente qui, en tant que telle, demeure toutefois constamment en dehors de l'ordre normal. " (1997: 182)

Si cette approche permet de mettre en évidence certaines spécificités du " gouvernement » des camps et de leurs caractéristiques, elle ne permet pas de saisir la nature plus complexe et ambiguë de nombreux camps de réfugiés palestiniens au Proche-Orient, et particulièrement les processus à travers lesquels les camps sont en même temps des lieux « exceptionnels » et " ordinaires ». Plusieurs auteurs ont récemment argumenté qu'il est nécessaire d’aller “ au-delà » d'Agamben (par exemple Ramadan 2012 ; Martin 2015) et d'étudier les camps dans leur complexité. C'est le point de vue adopté et poursuivi dans ce volume. Si à l'opposé de l'idée de l'espace d'exception se trouve le quartier urbain pleinement intégré à la cité, alors les différents camps de réfugiés palestiniens au Proche-Orient se trouvent répartis, selon leurs spécificités, à des endroits variés le long du spectre allant de l'un à l'autre.

La question du " temps ", de la durée, est un autre élément essentiel dans l'étude des camps. Parce que celle-ci transforme l'espace des camps (Agier 2001). Mais surtout, parce que, malgré le fait que certains camps de réfugiés palestiniens existent depuis plus de 60 ans, ils ont officiellement toujours un statut temporaire, de quasilieu d'attente, en attendant une résolution de la question des réfugiés dans le conflit israélo-palestinien. L’argument de cette publication est ainsi que les camps de réfugiés palestiniens au Proche-Orient sont tous marqués par ce " provisoire qui dure ", mais que les impacts de cette situation varient en fonction de chaque camp, selon de nombreux facteurs, par exemple le contexte national dans lequel il se trouve, 
son positionnement géographique, etc. Il s'agit donc d'une étude " comparative ", à plusieurs niveaux, des impacts que ce " provisoire qui s’installe dans la durée » a sur la population des camps.

Ce volume s’inscrit à la suite de plusieurs publications récentes de réflexions collectives qui ont traité, en totalité ou en partie, des réfugiés palestiniens au Proche-Orient, en abordant également la question des camps (par exemple Bocco et Takkenberg 2010 ; Knudsen et Hanafi 2011; Al Husseini et Signoles 2011 ; Doraï et Puig 2012 ; Hanafi et al. 2014). Ces publications portent sur les espaces de la diaspora palestinienne, sur l'évolution de la gouvernance des réfugiés palestiniens et des camps, et sur les dynamiques urbaines des camps et des réfugiés palestiniens dans les villes du Proche-Orient. Parallèlement, la question du temps, de la durée, et de l'institutionnalisation du provisoire qui n'en finit jamais comme condition de vie d'une part croissante de migrants dans le monde a fait l'objet d'une attention particulière dans les études récentes sur les migrations (voir Vosko et al. 2014). Nous proposons ainsi de poursuivre les débats initiés dans ces différents ouvrages, en réfléchissant explicitement à la question du temporaire qui se prolonge, et qui a tendance à se normaliser (Jamal 2009), et sur ses différents impacts politiques, socioéconomiques et urbains affectant les camps et les réfugiés palestiniens au cours de la période récente ${ }^{2}$.

\section{L'institution du provisoire qui dure}

Les premiers camps de réfugiés palestiniens ont été installés au Proche-Orient à la fin des années 40, à la suite de la création de l'État d'Israël. Les Palestiniens dénomment cet événement « Nakba » (signifiant « catastrophe »). Le 14 mai 1948, l'Agence juive à Tel-Aviv a annoncé l'institution du gouvernement provisoire d'Israël. La guerre qui s'en est suivie a engendré la destruction et l'évacuation en masse de la plupart des localités palestiniennes. 750'ooo personnes, dont de nombreuses blessées au cours du violent conflit arabo-israélien, ont d'un jour à l'autre été transformées en une question humanitaire. Les Palestiniens provenant du sud de la Palestine mandataire ont fui en direction de la Bande de Gaza, ceux provenant du centre se sont dispersés en Cisjordanie, puis en Jordanie, et ceux provenant du nord se sont dirigés au sud du Liban et de la Syrie (Kimmerling et Migdal 2003). Selon la Croix-Rouge, environ 320'00o Palestiniens sont restés en Cisjordanie, 210'ooo sont allés dans la Bande de Gaza, et 180'ooo sont partis en direcvolume concentrent tion d'autres pays arabes, dont 100'ooo ont trouvé refuge sur le territoire de la Jordanie contemporaine (Zureik 1996). principalement leurs analyses sur la première décennie du XXI ${ }^{\mathrm{e}}$ siècle. 
Bien que les États hôtes (Jordanie, Liban et Syrie) et le réseau d'organisations humanitaires impliqué dans l'aide aux réfugiés palestiniens avaient des mandats, des modes de fonctionnement et des intérêts différents, voire contradictoires, tous se sont dirigés vers une même réponse à la question palestinienne, à savoir la réinstallation. Deux a priori majeurs ont guidé ce régime, à savoir, premièrement, que les réfugiés palestiniens étaient un phénomène transitoire de crise, dont il ne faudrait que temporairement s'occuper, et deuxièmement, que la nature humaine devait être accommodée de façon sédentaire (Latte-Abdallah 2006). Dans ce contexte, les camps de réfugiés ont été prévus à l'attention des groupes de réfugiés paupérisés qui

6 s'étaient dispersés et n'avaient pas les moyens de se procurer un logement décent. Leur installation dans des camps bien organisés a permis d'améliorer l'acheminement de l'aide humanitaire et de réduire les coûts opérationnels. Les autorités locales et l'UNRWA pouvaient également, en les confinant dans un même espace, effectuer un meilleur contrôle d'une population potentiellement déstabilisatrice caractérisée par son attachement à la Palestine.

Les camps de réfugiés palestiniens n'ont pas été établis en tant qu'espaces d'exception où un " surplus de l'humanité » devait être tenu à l'écart de la population locale. Au contraire, le mandat initial de l'UNRWA, tel que défini par la résolution 302 (IV) de l'Assemblée générale des Nations Unies en décembre 1949, allait dans le sens d'une intégration rapide des réfugiés dans le marché du travail local et régional à travers un remplacement progressif de l'assistance par des programmes de travaux

3 Le 8 décembre 1949, l'Assemblée générale des Nations Unies (AGNU) a institué l'Office de secours et de travaux des Nations unies pour les réfugiés de Palestine dans le ProcheOrient (UNRWA) en tant qu'agence opérationnelle et non-politique devant répondre aux besoins humanitaires des réfugiés palestiniens à Gaza, en Cisjordanie, en Jordanie, en Syrie et au Liban. Selon la résolution 302 (IV) de décembre 1949 de l'AGNU, l'UNRWA devait travailler " in collaboration with local governments [on] the direct relief and works programmes [...] [and] consult with the interested Near Eastern Governments concerning measures to be taken by them preparatory to the time when international assistance for relief and works projects is no longer available " (AGNU 1949). publics, tels que la construction de routes et de systèmes d'irrigation, des travaux de boisement et de terrassement ${ }^{3}$. Les camps étaient la cible principale de la politique d'intégration de l'UNRWA. Il était prévu qu'ils soient démantelés et que la population soit relogée dans les villes et villages des alentours (Al-Husseini 2008).

Cependant, les tentes de toile qui avaient initialement été fournies aux réfugiés se révélaient vulnérables à l'usure du temps et ont progressivement été remplacées par des abris en dur. En raison de la nécessité de préserver la nature temporaire des camps, les gouvernements hôtes et l'UNRWA ont toutefois édicté des normes et des régulations 
spécifiques pour l'utilisation des parcelles de terrain et des abris dans les camps. Une unité d'habitation de 100 mètres carrés a été fournie à chaque famille possédant un numéro d'enregistrement certifiant son statut de réfugié. Sur chaque parcelle, un abri en dur comportant une pièce de 12 à 20 mètres carrés a été édifié. Comme les réfugiés ne pouvaient pas acquérir la propriété de la parcelle de terrain, celle-ci n'était en principe pas divisible ou transférable. Afin d'empêcher une croissance horizontale du camp, la construction de nouvelles unités était interdite. La hauteur des habitations était limitée à 3 mètres afin de limiter l'élévation du camp. Les résidents des camps n'avaient pas la permission de louer, d'acheter ou de vendre leur unité d'habitation. Ainsi, à ses débuts, la vie dans les camps était disciplinée en limitant l'utilisation de l'espace au logement et en empêchant toute autre affectation (Destremau 1994 ; Jaber 1996). De plus, les camps ont été tenus à l'écart des planifications nationales et municipales en matière de développement urbain. Une détermination à reproduire le caractère temporaire des camps se trouvait à la base de ces principes. Les réfugiés n'étaient pas opposés à ces choix puisqu'ils craignaient eux-mêmes qu'un développement des infrastructures signifie la remise en question de leur retour en Palestine. Ainsi, jusqu'aux années 6o, les camps sont restés déconnectés des réseaux d’infrastructures publiques (eau, électricité, lignes téléphoniques) des municipalités adjacentes (Destremau 1994 ; Al-Husseini 2011).

\section{L'évolution et l'appropriation des camps}

Plusieurs facteurs, incluant la faible capacité d'absorption des pays hôtes et l'absence d'une solution politique à la question des réfugiés, ont compliqué la réinstallation des réfugiés. Plus important encore, les programmes de travail de petite et grande importance mis en place dans les années 50 par l'UNRWA, et visant à une réintégration, ont rencontré une importante opposition de la part des réfugiés. La notion d' "implantation " (tawtin) a été interprétée comme un signe de collaboration avec Israël, et les camps sont devenus des symboles politiques de la lutte palestinienne pour le droit au retour (Meier 2009).

Au fur et à mesure, les camps de réfugiés se sont mués en des espaces temporaires à préserver. Ils sont devenus des lieux de référence où les résidents ont reconstruit leur identité, leur histoire, et leurs conceptions sociale, culturelle et politique. De nombreux intellectuels ou artistes palestiniens ont été inspirés par les camps en tant que symboles de l'expérience de la vie en exil (Sayigh 1998). Plusieurs auteurs ont également analysé la façon dont les Palestiniens se sont appropriés l'espace des camps et ses symboles, en créant par exemple des établissements commerciaux 
illégaux dans leurs habitations, en squattant les bâtiments gouvernementaux à des fins politiques, ou en faisant des documents d'identité établis par l'UNRWA des emblèmes de la résistance politique contre l'occupation israélienne de la Palestine (voir par exemple Bisharat 1997 ; Marx 1992 ; Peteet 2005 ; Sayigh 1998).

Les camps de réfugiés au Proche-Orient ont une longue histoire de résistance politique et de mobilisation nationale. De plus, les réfugiés se sont opposés à l'UNRWA lorsqu'ils suspectaient celle-ci d'entreprendre des mesures conduisant à une « implantation ». Par exemple, en Jordanie, le camp d'al-Wahdat à Amman est

8 devenu un lieu central des activités nationalistes palestiniennes à partir du début des années 6o, et ceci jusqu'au début des années 70 . En dix ans, il a presque atteint une entière autonomie politique par rapport au pouvoir central de l'État jordanien. À la veille de la guerre civile jordanienne en 1970, connue sous le nom de « Septembre noir ", al-Wahdat n'était pas seulement une base pour les militants nationalistes palestiniens, mais un véritable centre de commandement militaire. Le mouvement de libération de la Palestine, principalement des membres des organisations du Fatah et du Front populaire de libération de la Palestine (FPLP), y avait établi leur quartier général, et le camp fut renommé « La République » en signe de défiance à la Monarchie jordanienne. Même après la désintégration des groupes armés de Fedayins à la suite de la guerre civile, le camp d'al-Wahdat a conservé son caractère politique et culturel distinct.

Les camps qui étaient des instruments de subjectivation des réfugiés et des symboles de leur cause politique et humanitaire sont devenus le socle de la formation d'une identité nationale dans un contexte d'exil prolongé. À travers le temps, les manifestations propalestiniennes, les protestations et les symboles associés à la vie dans les camps, par exemple la carte de réfugié, les plaques métalliques utilisées pour couvrir les abris, ou encore le drapeau des Nations Unies, sont restés pour les réfugiés des actes et des marqueurs importants d'identification à la cause nationale palestinienne. Cependant, si les temps héroïques des années 60 et 70 ont joué, et dans une certaine mesure jouent toujours, une place centrale dans la conscience politique des réfugiés, les camps ont, au fil des années, perdu une grande part de leur réputation de bastions de la résistance palestinienne.

Alors qu'ils avaient été au centre du mouvement national palestinien, les réfugiés palestiniens et les camps au Proche-Orient se sont retrouvés en marge du processus politique. En reléguant le statut des réfugiés aux étapes finales des négociations 
du processus de paix israélo-palestinien, les accords d'Oslo de 1993 ont été pour les réfugiés palestiniens au Liban, en Syrie et en Jordanie, un signe évident que l'Autorité palestinienne en formation avait bradé leur droit au retour afin d'assurer la construction d'un État palestinien (Tamari 1996)4 . Leur exclusion du projet national officiel a engendré auprès des réfugiés de l'amertume et de la frustration envers l'activisme politique. Ce désengagement de la participation politique a été motivé également par d'autres facteurs, notamment les restrictions des activités politiques imposées par les autorités hôtes, le désenchantement engendré par la rivalité entre les organisations du Hamas et du Fatah5, et l’inefficacité des partis défendant la cause palestinienne.

Aujourd'hui, le manque d'opportunités de travail et la pauvreté dans les camps ont contribué à renforcer le sentiment parmi les résidents des camps, ainsi que parmi les sociétés hôtes plus généralement, que les camps de réfugiés sont des lieux affectés par de nombreux problèmes, allant de la marginalisation socioéconomique, à la criminalité et au radicalisme islamique (Hamarneh 2002 ; Rougier 2006). Par ailleurs, en plus de la transformation informelle de l'espace des camps par les résidents, se traduisant souvent par un agrandissement vertical, voire horizontal, des habitations, au-delà des limites prévues, des projets de réhabilitation urbaine des camps ont également progressivement été mis en place, de façon plus ou moins officielle et formelle. En Jordanie, les camps ont par exemple été inclus dans un programme national d'amélioration des infrastructures à la fin des années 9o. Ces projets ont contribué à une intégration progressive des camps aux espaces urbains environnants, les camps ressemblant de plus en plus à des quartiers populaires. Ils se sont aussi retrouvés habités par une population de plus en plus mixte et non exclusivement palestinienne. Cependant, si les camps ont ainsi souvent des frontières invisibles ou floues, ils gardent en revanche des limites administratives rigides. De plus, ils conservent et partagent souvent des ressemblances qui leur confèrent un caractère distinct, telles les étroites ruelles qui leur donnent des allures de labyrinthe, ainsi que la relative homogénéité des constructions et de la hauteur des bâtiments. Il est également possible de remarquer d'autres marqueurs persistants de différenciation dans l'esthétique du camp, par exemple l'emblème des Nations Unies

4 Alors que les accords d'Oslo ont
relégué les réfugiés et les camps
en marge du discours nationaliste
palestinien, il faut noter qu'en
dissipant les espoirs de paix, l'Inti-
fada Al-Aqsa en 2000 les a en partie
ramené au centre de l'arène poli-
tique (voir Khalili 2007 : $57-58$ ).
5 En 20o6, les tensions entre les
deux principaux partis politiques
palestiniens (le Hamas et le Fatah)
ont débouché sur la création de
deux gouvernements séparés, à
savoir le gouvernement du Hamas
dans la Bande de Gaza et l'Autorité
palestinienne dirigée par le Fatah
en Cisjordanie.

4 Alors que les accords d'Oslo ont relégué les réfugiés et les camps en marge du discours nationalist dissipant les espoirs de paix, l'Intifada Al-Aqsa en 2000 les a en partie ramené au centre de l'arène poli5 En 2006, les tensions entre les deux principaux partis politiques palestiniens (le Hamas et le Fatah) ont débouché sur la création de deux gouvernements séparés, à dans la Bande de Gaza et l'Autorité en Cisjordanie. 
apposé contre certains bâtiments officiels, ainsi que les écoles et autres établissements de l'UNRWA.

Au final, la politisation des camps a contribué à assurer leur existence, tout en respectant leurs caractéristiques spécifiques. Les camps sont ainsi devenus des lieux à préserver, des sanctuaires d'une " palestinité ". Ils sont des symboles grâce auxquels les réfugiés peuvent rappeler à la communauté internationale l’absence d'une solution politique à leur cause, et exprimer un sentiment partagé d'unité nationale.

\section{Problématiser le provisoire qui dure : la Jordanie et le Liban}

Malgré leur évolution, les camps de réfugiés palestiniens au Proche-Orient demeurent des espaces temporaires. La gestion et l'organisation des camps reflètent cette particularité. Ils ne sont pas gérés par les municipalités à l'intérieur desquels ils sont situés, mais par, d'un côté, l'UNRWA et, d'un autre côté, des institutions gouvernementales des pays hôtes (Jordanie et Syrie), ou des organisations politiques palestiniennes (Liban et Territoires palestiniens occupés). L’UNRWA est principalement en charge de la fourniture de services (éducatifs, sociaux et de santé).

Au-delà de ce socle commun, les habitants des camps se retrouvent dans des conditions très différentes, variant principalement en fonction des contextes nationaux. Par exemple, alors que les réfugiés palestiniens sont également depuis 1949 des citoyens en Jordanie, la majorité de ceux résidant dans les autres pays du Proche-Orient demeure "sans-État ». Les réfugiés ont également été sujets de systèmes variés de discriminations. S’il existe une quasi-parité en Jordanie où les réfugiés bénéficient des pleins droits de citoyenneté, ils doivent faire face à des discriminations invisibles (par exemple au niveau des emplois dans le secteur public, de la représentation politique, etc.), alors qu'ils sont soumis à une marginalisation complète et avérée au Liban. Au niveau de l'espace des camps, cela se traduit par, selon les termes de Sari Hanafi (2009), soit des camps " ouverts " (Jordanie et Syrie) et connectés à l'espace urbain environnant, soit des camps « fermés » (Liban et Territoires palestiniens occupés) représentant des enclaves urbaines, bien que les frontières puissent demeurer floues selon les cas.

Ainsi, afin d'étudier les impacts politiques, socioéconomiques et spatiaux différenciés du provisoire qui dure, les contributions de ce volume se concentrent sur deux cas relativement distincts et opposés, à savoir la Jordanie d'un côté, et le Liban d'un autre. En tenant compte de la complexité associée à l'espace des camps et à 
l'expérience de l'exil, les auteurs se concentrent sur la question de savoir comment le temporaire est expérimenté et négocié par les habitants des camps ou ceux qui sont en charge de leur gestion. Les camps ne sont ainsi pas perçus comme des territoires clos d'une communauté palestinienne figée, mais comme des creusets et des lieux de changements, de négociations et de conflits. C'est à travers ces perspectives multiples, et l'analyse à la fois des dynamiques transversales et des particularités qu'il sera possible de mieux comprendre les évolutions des camps de réfugiés palestiniens au Proche-Orient. Nous espérons que ces éclairages peuvent également servir à l'analyse plus générale des espaces des migrations forcées au-delà de cette région.

Les articles de ce volume vont ainsi aborder plusieurs thèmes essentiels à cette compréhension. En premier lieu, il est question de la mise en place et de la cristallisation du provisoire dans les camps, et de ses effets.

À travers une analyse des camps de réfugiés palestiniens en Jordanie, l'article de Luigi Achilli et Lucas Oesch montre que, afin de rendre compte de la multidimensionnalité qui caractérise l'expérience des réfugiés dans les camps, il est nécessaire de s'intéresser aux processus complexes par lesquels les camps deviennent des lieux " exceptionnels », mais aussi " ordinaires ». Les auteurs argumentent que le fonctionnement des camps en tant qu' " espaces d'ambiguïté " est le concept qui permet le mieux de saisir leur nature. En Jordanie, c'est le caractère ambigu des camps de réfugiés palestiniens qui permet de garantir une forme de stabilité politique. Afin de mettre en évidence cette ambiguïté, après avoir étudié son origine et sa mise en place, l'article analyse sa spatialisation dans les camps, en s'intéressant à la gestion de ces lieux. Il montre que la re-production de cette ambiguïté est le fait des acteurs en charge des camps, ainsi que des réfugiés eux-mêmes.

En deuxième lieu, les articles abordent les questions de l'organisation politique et de la gouvernance dans les camps.

L'article de Sergio Bianchi montre que le camp est une lentille distordante des études sur les réfugiés palestiniens, ces dernières ayant tendance à essentialiser les conditions de l'exil palestinien à celles des camps. Par une analyse des « tours guidés » des camps au Liban, il s'intéresse ensuite aux processus de production de connaissances à propos des réfugiés palestiniens. En examinant les relations entre les différents acteurs impliqués dans ces visites (les visiteurs, les accompagnateurs et les visités), il met en évidence les rapports de force et les compétitions qui existent 
entre les différentes représentations symboliques des camps et de leurs habitants. Il s'agit de luttes de pouvoir ayant des effets concrets sur les processus de gouvernance des camps, puisqu'elles visent au contrôle du camp et à l'appropriation des ressources provenant de l'aide internationale. En dernier lieu, l'auteur souligne le manque de légitimité que cette production de savoir obtient auprès des habitants des camps, une carence qui est, selon lui, à la base d'une crise plus généralisée des institutions palestiniennes.

L'article de Hala Abou Zaki met également en évidence les processus complexes 12 de gouvernance et d'organisation politique dans les camps. Par l'analyse des conditions de la création, puis du déclin, d'un nouveau comité des habitants dans un camp libanais, elle montre que les camps sont investis par une multitude de pouvoirs qui ont des ramifications locales, nationales, régionales et internationales. Ils coexistent et luttent pour le contrôle du camp et entravent les possibilités d'autoorganisation populaire et locale. L’auteure souligne aussi que les visées du comité étaient limitées à l'amélioration des conditions de vie et des services dans le camp, et que malgré cette focalisation le comité est entré en compétition avec d'autres organes de représentation des camps. Les infrastructures « techniques " sont ainsi des outils politiques, et l'amélioration des camps est également une lutte pour le pouvoir et les ressources.

En troisième lieu, les articles de ce volume reviennent sur les questions du développement urbain, de l'habitat et des conditions de vie dans les camps et leurs alentours.

À travers l'étude de l'extension du camp de Nahr el-Bared, l'article de Rana Hassan montre comment ces lieux informels représentent un potentiel de production de logements pour les réfugiés palestiniens vivant en marge des limites des camps au Liban. Ainsi, ils peuvent dans certains cas bénéficier de meilleures conditions de vie que les habitants des camps. Cependant, les habitants de ces lieux informels sont plus exposés aux lois qui les marginalisent, et peuvent donc être plus vulnérables dans certaines circonstances. Dans le cas de la reconstruction de l'extension de Nahr el-Bared après la guerre de $\mathbf{2 0 0 7}$, le gouvernement libanais a ainsi choisi d'utiliser la légalité comme outil de répression et de contrôle de la zone, afin d'y réaffirmer sa souveraineté. L'article de Rana Hassan remet ainsi en question le concept d'espace d'exception, en argumentant que si les camps au Liban isolent les Palestiniens, et les excluent des droits et avantages dont ils devraient bénéficier en tant que résidents 
du Liban, il est aussi possible de faire valoir qu'ils servent à les protéger de la stricte application par les représentants de l'État libanais des lois et règlements qui les marginalisent.

L'article de Myriam Ababsa montre, à travers une analyse cartographique et statistique, que les camps de réfugiés en Jordanie partagent certaines caractéristiques démographiques et socioéconomiques avec les quartiers informels et populaires qui les entourent, eux-mêmes habités en grande partie par des réfugiés palestiniens. Elle remarque que les camps palestiniens à Amman ont, en plus, été progressivement intégrés physiquement au tissu urbain municipal, par les réseaux d'adduction en eau, les égouts et l'électricité, particulièrement après le processus de paix de 1993 et les accords israélo-jordaniens de Wadi Araba (octobre 1994). Elle conclut que malgré ces analogies, les camps conservent une identité particulière, liée à leur signification politique. D’après les mots de l'auteur, les camps symbolisent la non-résolution du conflit israélo-palestinien et la responsabilité de la communauté internationale. Mais ils sont aussi le symbole du contrat social jordanien selon lequel les réfugiés palestiniens sont des citoyens jordaniens et les rois de Jordanie sont garants de leur droit au retour.

Au final, les articles de ce volume mettent en évidence que « le provisoire qui dure » ne fait pas des camps de réfugiés palestiniens au Proche-Orient des espaces d'exception figés, que ce soit au niveau de leur gouvernance ou organisation politique, de leurs caractéristiques urbaines, démographiques et socioéconomiques, ou encore au niveau de l'engagement politique de leurs habitants. Le statut de ces espaces est donc beaucoup plus mouvant, que ce soit dans le cas de la Jordanie où la marginalisation des habitants des camps est ambiguë, et même dans le cas du Liban où celle-ci est beaucoup plus avérée. À travers les exemples de la Jordanie et du Liban, et leurs effets de miroir, les articles révèlent que les différents contextes nationaux conduisent à des configurations variées, ce qui a tendance à renforcer encore plus l'argument que les camps de réfugiés sont des espaces dynamiques, contextuels et modulables. 


\section{Références}

AGAMBEN Giorgio (1997), Homo sacer I : Le pouvoir souverain et la vie nue, Paris, Seuil. AGAMBEN Giorgio (2003), Homo Sacer II : État d'exception, Paris, Seuil.

AGIER Michel (2001), " De nouvelles villes : les camps de réfugiés ", Les Annales de la Recherche Urbaine, $\mathrm{N}^{\circ}$ 91, pp. 128-136.

AGIER Michel (2008), Gérer les indésirables : des camps de réfugiés au gouvernement humanitaire, Paris, Flammarion, Bibliothèque des savoirs.

14 AGNU (1949), General Assembly Resolution 302, A/RES/302 (IV), 8 December 1949. URL : www.unrwa.org/content/general-assembly-resolution-302, consulté le 4 septembre 2014 .

AL-HAMARNEH Ala (2002), " The Social and Political Effect of Transformation Processes in Palestinian Refugee Camps in the Amman Metropolitan Area (1989-99) ", in Jordan in Transition 1990-2000, G. Joffe (dir.), London, Urst \& Company.

AL-HUSSEINI Jalal (2008), « Les camps de réfugiés palestiniens au Proche-Orient, entre norme du droit au retour et intégration socioéconomique ", Asylon(s), $\mathrm{N}^{\circ} 5$. URL : www.reseau-terra.eu/article79g.html, consulté le 4 septembre 2014.

AL-HUSSEINI Jalal (2011), «The Evolution of the Palestinian between Logics of Exclusion and Integration ", in Villes, pratiques urbaines et construction nationale en Jordanie, M. Ababsa et R. Daher (dir.), Beirut, Presses de l'IFPO.

AL-HUSSEINI Jalal et Aude SIGNOLES (dir.) (2011), Les Palestiniens entre Nation et Diaspora. Le temps des incertitudes, Paris, IISMM et Karthala.

BISHARAT George (1997), "Exile to Compatriot : Transformation in the Social Identity of Palestinian Refugees in the West Bank ", in Culture, Power, Place : Exploration in Critical Anthropology, A. Gupta et J. Ferguson (dir.), London, Duke University Press.

BOCCO Riccardo et Lex TAKKENBERG (dir.) 2010, " UNRWA and the Palestinian Refugees 60 Years Later ", Refugee Survey Quarterly, vol. 28, N²-3.

DANIEL Valentine et John KNUDSEN (dir.) (2011), Mistrusting Refugees, Berkeley/London, University of California Press.

DESTREMAU Blandine (1994), "L'espace du camp et la production du provisoire : les camps de réfugiés palestiniens de al-Wihdat at Jabal Hussein à Amman ", in MoyenOrient, Démocratisation, Médiation, R. Bocco et M. Djalili (dir.), Paris, PUF.

DORAÏ Mohamed Kamel et Nicolas PUIG (2012), L’urbanité des marges : Migrants et réfugiés dans les villes $d u$ Proche-Orient, Paris, Téraèdre.

HANAFI Sari (2009), " Palestinian Refugee Camps in the Palestinian Territory: Territory of Exception and Locus of Resistance ", in The Power of Inclusive Exclusion: 
Anatomy of Israeli Rule in the Occupied Palestinian Territories, A. Ophir, M. Giovanni et S. Hanafi (dir.), New York, Zone Books, pp. 495-517.

HANAFI Sari, Leila HILAL et Lex TAKKENBERG (dir.) (2014), UNRWA and Palestinian Refugees : From Relief and Works to Human Development, London, Routledge.

HARRELL-BOND Barbara (1999), " The Experience of Refugees as Recipient of Aid ", in Refugees. Perspectives on the Experience of Forced Migration, A. Ager (dir.), London, Continuum.

HYNDMAN Jennifer (2000), Managing Displacement : Refugees and the Politics of Humanitarianism, Minneapolis, University of Minnesota Press.

JABER Hana (1996), «Le camp de al-Wihdat, entre norme et transgression », Revue d'études palestiniennes, $\mathrm{N}^{\circ}$ 8, pp. 37-48.

JAMAL Amal (2009), "The Struggle for Time and the Power of Temporariness : Jews and Palestinians in the Labyrinth of History ", in Men in the Sun, T. Ben Zvi et H. Farah-Kufer Bir'im (dir.), Herzliya, Herzliya Museum of Contemporary Art.

KHALILI Laleh (2007), Heroes and Martyrs of Palestine: The Politics of National Commemoration, Cambridge, Cambridge University Press.

KIMMERLING Baruch et Joel MIGDAL (2003), The Palestinian People : A History, Cambridge, Harvard University Press.

KNUDSEN Are et Sari HANAFI (dir.) (2011), Palestinian Refugees : Identity, Space and Place in the Levant, London/New York, Routledge.

LATTE-ABDALLAH Stéphanie (2006), Femmes réfugiées palestiniennes, Paris, PUF.

LONG Lynellyn (1993), Ban Vinai : the Refugee Camp, New York, Columbia University Press.

LOW Setha et Denise LAWRENCE-ZUNIGA (dir.) (2002), The Anthropology of Space and Place: Locating Culture, Oxford, Blackwell.

MALKKI Liisa (1995), Purity and Exile: Violence, Memory, and National Cosmology among Hutu Refugees in Tanzania, Chicago, University of Chicago Press.

MALKKI Liisa (1997), " National Geographic : the Rooting of Peoples and the Territorialization of National Identity among Scholars and Refugees ", in A. Gupta et J. Ferguson (dir.), Culture, Power, Place : Exploration in Critical Anthropology, London, Duke University Press.

MARTIN Diana (2015), « From Spaces of Exception to <Campscapes> : Palestinian Refugee Camps and Informal Settlements in Beirut ", Political Geography, vol. 44, pp. 9-18.

MARX Emanuel (1992), "Palestinian refugee camps in the West Bank and the Gaza Strip ", Middle Eastern Studies, vol. 28, N² 2, pp. 281-294.

MEIER Daniel (2009), "<L'implantation> comme construction de la présence palestinienne au Liban durant la tutelle syrienne (1989-2005)", Revue des mondes musulmans et de la Méditerranée, $\mathrm{N}^{\circ} 126$, pp. 223-242. 
\{ Introduction

PETEET Julie (2005), Landscape of Hope and Despair : Palestinian Refugee Camps, Philadelphia and Bristol, University of Pennsylvania Press.

RAMADAN Adam (2012), "Spatialising the Refugee Camp ", Transactions of the Institute of British Geographers, vol. 38, N ${ }^{\circ}$, pp. 65-77.

ROUGIER Bernard (2007), Everyday Jihad: The Rise of Militant Islam among Palestinians in Lebanon, London, Harvard University Press.

SAYIGH Rosemary (1998), " Dis/Solving the refugee problem », Middle East Report, vol. 207, pp. 19-23.

SOGUK Nevzat (1999), States and Strangers : Refugees and Displacements of Statecraft, Minneapolis, University of Minnesota Press.

16 TAMARI Salim (1996), Palestinian Refugee Negotiations : From Madrid to Oslo II Washington, D.C., Institute for Palestine Studies.

UNRWA (2015), UNRWA in Figures (as of 1 January 2015), Jerusalem, Communications division, UNRWA Headquarters.

VOSKO Leah, Valerie PRESTON et Robert LATHAM (dir.) (2014), Liberating Temporariness? Migration, Work, and Citizenship in an Age of Insecurity, McGill, Queen's University Press.

ZUREIK Elias (1996), Palestinian Refugees and the Peace Process, Washington D.C., Institute for Palestine Studies. 